\title{
WORK-HOME INTERACTION OF EMPLOYEES \\ IN THE MINING INDUSTRY
}

K Mostert and G R Oldfield

WorkWell: Research Unit for People, Policy and Performance North-West University

\begin{abstract}
This study aimed to test the construct validity, factorial invariance and reliability of the Survey Work-Home Interaction-NijmeGen (SWING) and to explore whether and how the work-home interaction of various socio-demographic groups differ. Random samples $(n=320)$ were taken of employees in the mining industry. The confirmatory factor analysis results supported the proposed four-factor structure measuring negative/positive work-home interference and negative/positive home-work interference. The multi-group invariance analyses' results for two language and ethnic groups also supported the factorial invariance of the SWING. All the scales were found to be reliable. Statistically significant differences in work-home interaction were found, based on age, ethnicity, gender, education, marital status, parental status, language, flexibility at work and individuals who had a partner with a paid job.
\end{abstract}

JEL J24, J28

\section{1}

\section{Introduction}

Because work and family constitute the dominant life roles for most employed adults in contemporary society, employed men and women are increasingly concerning themselves with managing the conflicts they experience in attempting to fulfil the dual demands and responsibilities of work and family roles. According to Greenhaus and Beutell (1985), work-family conflict is experienced when pressures from the work and family roles are mutually incompatible, in the sense that participation in one role makes it difficult to participate in the other. The result is that individuals may experience some form of conflict between the roles they assume they must fulfil and the roles they are expected to fulfil. Work-family issues are also believed to affect company competitiveness and are therefore not only a problem for employees, but also for organisations (Allen, Herst, Bruck \& Sutton, 2000; Houston, 2005; Lewis \& Cooper, 2005; Parasuraman \& Greenhaus, 1999).
In South Africa, increased attention to the work-home nexus could be attributed to the increase in dual-earner couples and the fact that the South African economy has opened up, and that females are increasingly hired, ensuring equity and a socio-demographically representative workforce. This is also true for the mining industry.

People employed in the mining industry have to face various demands and often unpleasant working conditions (Calitz, 2004). They usually have to work shifts, which deprives them of time they can spend with family and friends during "normal" social hours. They may also be required to work in dark and damp conditions at varying temperatures (Singer, 2002), usually deep underground, and often have to work alone in small areas with little supervision and communication (Calitz, 2004). The mining industry also has the highest rate of fatal occupational injuries: more than one hundred miners are killed in the South African mining industry every year (McGwin et al, 2002). As a result, the Chamber of Mines had to develop safety systems management programmes 
within mines to assist employees in coping effectively with the demands and pressures that adversely affect their quality of life, health and productivity after traumatic mining incidents (Badenhorst \& Van Schalkwyk, 1992; Chamber of Mines of South Africa, 1989). This type of working environment poses a challenge to people who want to integrate their work and family lives successfully.

Although the work-home nexus can be regarded as an important area to study, research in this field is characterised by two major limitations. Firstly, the majority of empirical studies focus on the negative interference between work and personal life and they base their hypotheses on the role scarcity hypothesis (which assumes that people possess limited and fixed amounts of resources like time and energy). This implies that positive work-home interaction and the idea of role enhancement (which proposes, for example, that fulfilling multiple roles may produce resources such as energy mobilisation, skill acquisition and greater self-esteem, which facilitate functioning in both life spheres) have been under-researched. Secondly, many instruments are available to measure negative work-home interaction, as opposed to only a few instruments exclusively developed for measuring positive interaction the instruments developed by Carlson, Kacmar, Wayne and Grzywacz (2006) and Kirchmeyer (1992) are among the few that look at positive interactions. Instruments developed for the measurement of both negative and positive interaction are even more rare.

In South Africa, research on the measurement of work-home interaction is scarce, and so far, there is no instrument that has been proven to be valid and reliable in measuring work-home interaction within the mining industry. Due to the nature and diversity of the mining industry, it is also important to have an instrument that can be used across diverse groups. Therefore, an instrument is needed that is also invariant for different language and ethnical groups.

An instrument called the Survey Work-Home Interaction-NijmeGen (SWING) was developed and validated by Geurts et al (2005) at the Radboud University in the Netherlands. What makes this instrument unique is the fact that that it gives a full theory-guided conceptualisation of the work-home interface and encompasses interaction between both direction (interaction between the work domain and the home domain) and quality (negative and positive interaction). As a result, it captures the negative as well as the positive dimensions of the interaction between work and home.

In South Africa, only two studies have been found that have to date investigated the psychometric properties of the SWING (Pieterse \& Mostert, 2005; Rost \& Mostert, 2007). These studies found the SWING to be a valid, invariant and reliable measuring instrument. However, although these findings are encouraging, it could not be assumed that the findings can be applied to the mining industry. It was therefore necessary to investigate the psychometric properties of the SWING in a sample of mining employees before valid and reliable conclusions could be drawn regarding work-home interaction in the South African mining industry. Replication of the construct validity of the SWING was therefore important because, firstly, it would provide assurance that the results obtained in the previous studies are valid and reliable for use in the mining industry; and, secondly, it would investigate the generality of previous results.

In any organisation, there are several challenges in and strategies for managing demographic diversity. According to Tsui and Gutek (1999), two ways of managing diversity are recognised: firstly, attending to the social psychological processes that mediate relations between demographic characteristics and social and performance outcomes and, secondly, managing the distribution of workers on the basis of demographic characteristics.

Information regarding the relationship between socio-demographic characteristics and work-home interaction are very rare in the South African literature, and no information could be found on whether and how socio-demographic groups differ with regard to their work-home interactions in the mining industry. According to Eldridge and Miles (2008), accommodating and understanding demographic differences can give an organisation a competitive advantage and help organisations to meet the needs of 
their employees better, leading to higher levels of motivation, satisfaction and commitment. Therefore, in order for companies in the South African mining industry to identify possible risk groups that may struggle to balance their work and home lives, it seems important to determine whether or not there are differences regarding work-home interaction between different sociodemographic groups and to explore how these groups differ. This could assist companies to create a strategy that suits the organisational culture and is flexible enough to satisfy the needs and priorities of different groups of employees.

In view of the above discussion, the first objective of this study was to validate the psychometric properties (including the construct validity, factorial invariance and reliability) of the SWING against previous research results. The second objective was to explore differences between socio-demographic groups in the mining industry with regard to work-home interaction.

\subsection{The work-home interface}

Because work-home interaction has become increasingly important, the need for an instrument based on a sound theoretical background has become vital. To overcome the limitations which would be imposed by the absence of such a theoretical base, the SWING is based on a theoretical perspective called the Effort-Recovery (E-R) model (Meijman \& Mulder, 1998). The E-R model sheds light on how work and private life may interact and by which mechanisms well-being may be affected (Geurts, Kompier, Roxburgh \& Houtman, 2003). According to this model, effort expenditure is associated with specific load reactions (namely physiological, behavioural and subjective responses) that develop within the individual, such as changes in hormone secretion, energy levels and mood. These reactions are, in principle, reversible. Recovery takes place when the exposure to load ceases and the respective psychological systems stabilise again at a specific baseline level within a certain length of time (Drenth, Thierry \& De Wolff, 1998). However, when demands do not cease, no recovery occurs.
As a result, negative load effects develop, which may result in increased load reactions, which in turn make higher demands on the recovery process. Thus, a cumulative process may lead to a drain of energy and a state of breakdown or exhaustion (Sluiter, 1999; Ursin, 1980).

The fundamental role of the recovery process clearly makes the E-R model a promising perspective for studying negative work-home interaction. However, the same perspective may also increase our understanding of positive workhome interaction since effort expenditure may also be accompanied by positive load reactions. If a person feels competent and satisfied in his or her work, these positive feelings could translate to the home sphere (and vice versa).

Geurts et al (2005:322) based their definition of work-home interaction on the E-R model. They define work-home interaction as "an interactive process in which a worker's functioning in one domain (e.g. home) is influenced by (negative or positive) load reactions that have built up in the other domain (e.g. work)". Difficulties in combining work and family roles may arise from time demands that make it physically impossible to be in two places at the same time (time-based conflict), from the spill-over of strain from one domain to the other (strain-based conflict), or from the incompatibility of behaviours requested in each domain (behaviour-based conflict) (Greenhaus \& Beutell, 1985).

\subsection{The Survey Work-Home Interaction - Nijmegen (SWING)}

The SWING differentiates between the direction as well as the quality of interference between work and home. By measuring work-home interaction in this way, four factors are measured. The first is negative work-home interference (WHI) (when negative load reactions built up at work, hampering functioning at home). The second is positive WHI (when positive load reactions built up at work, facilitating functioning at home). The third is negative home-work interference (HWI) (when negative load reactions develop at home, impeding functioning at work). The fourth is positive HWI (when positive load reactions develop at home, facilitating functioning at work) (Geurts et al, 2005). 
In the study of Geurts et al (2005), nine items were designed to measure negative WHI (five items covering strain-based interference, and four items covering time-based interference). Negative HWI is measured by six items (including four self-developed items). Five of these items parallel items from the negative WHI scale. Positive WHI is measured by six items, of which five items were self-developed. Two items tap the spill-over of positive mood, while four items cover the transfer of skills learned at work. Positive HWI is measured by six items, of which five items were self-developed to parallel the five positive WHI items. Again, two items capture the spill-over of positive mood, while three items measure the transfer of skills learned at home (Geurts et al, 2005). Although the SWING originally consisted of 27 items, the final version of the questionnaire included 22 items, of which 13 items were newly developed.

By using data from five independent samples (total $N=2472$ ), Geurts et al (2005) provided evidence for the validity of the internal structure of the questionnaire. Their results showed that the questionnaire reliably measured four empirically distinct types of work-home interaction (as outlined above), and that this four-dimensional structure was largely invariant across the five independent samples, as well as across relevant subgroups, providing evidence regarding its robustness across a wide variety of workers. Similar results were obtained in two South African studies (Pieterse \& Mostert, 2005; Rost \& Mostert, 2007). Using principal component analysis with a direct oblimin rotation, Pieterse and Mostert (2005) obtained four factors in a sample of workers in the earthmoving industry. They also demonstrated construct equivalence for two language groups, although three problematic items had to be removed. Rost and Mostert (2007) also found that the four-factor structure of the SWING fitted the data significantly better than alternative models.

The four scales of the SWING show sufficient reliability. Geurts et al (2005) report Cronbach Alpha coefficients of 0.84 for negative WHI, 0.75 for positive WHI, 0.75 for negative HWI and 0.81 for positive HWI. Pieterse and Mostert
(2005) obtained the following Cronbach Alpha coefficients for the SWING: 0.87 for negative WHI, 0.79 for positive WHI, 0.79 for negative HWI and 0.76 for positive HWI. Rost and Mostert (2007) also obtained reliable Cronbach Alpha coefficients for the SWING dimensions ( 0.86 for negative WHI; 0.77 for positive WHI; 0.71 for negative HWI and 0.79 for positive HWI).

In summary, the present study proposes the following:

Hypothesis 1 The SWING has a four-dimensional structure (namely, negative WHI, positive WHI, negative HWI and positive HWI);

Hypothesis 2 The factor structure of the SWING is invariant for the two language and ethnic groups in this study; and

Hypothesis 3 The SWING has acceptable reliability coefficients.

\subsection{Work-home interaction and socio-demographic differences}

Since research regarding the relationship between socio-demographic differences and workhome interaction is limited and inconclusive (particularly in South Africa), the second part of the study was explorative in nature, and sought to answer the following research questions:

Research question 1 Are there differences between the work-home interaction levels of different socio-demographic groups?

Research question 2 If so, how do these groups differ with regard to the four work-home interaction dimensions (negative WHI, positive WHI, negative HWI and positive HWI)?

Socio-demographic variables included the following: age, ethnicity, gender, education, marital status, parental status, language, flexibility at work, if the participant has a partner with a paid job and the financial contribution of the partner to the household situation.

With regard to age, Grzywacz and Marks (2000) found that younger men reported a higher negative spill-over between work and home (as well as between home and work) and less positive spill-over from home to work than 
older men. They also found that younger women reported more positive spill-over from work to home and more negative spill-over from home to work than older women did. However, most other studies found no relationship between different age groups (Frone, Russell \& Cooper, 1997; Kinnunen \& Mauno, 1998; Pieterse \& Mostert, 2005).

Regarding ethnicity, Pieterse and Mostert (2005) found no differences between ethnic groups. However, Grzywacz and Marks (2000) found that black women reported less negative spill-over from home to work than other women did. In a sample of nurses, Van Tonder (2005) found statistically significant differences between Caucasian and African nurses regarding home-work interference: Caucasians experienced more negative HWI, but also more positive HWI.

Several studies revealed that there are hardly any differences between males and females in their experience of negative or positive interaction between work and home, in both directions (Burke, 1988; Demerouti, Geurts, Bakker \& Euwema, 2004; Eagle, Miles \& Icenogle, 1997; Frone, 2002; Kinnunen \& Mauno, 1998; Kirchmeyer, 1992). Therefore, no differences were expected between males and females. Frone et al (1997) found no significant relationships between educational level and work-home interference. However, Pieterse and Mostert (2005) and Van Tonder (2005) found significant differences between different educational groups: individuals with a Technicon diploma experienced a significantly higher negative WHI than individuals with a Grade 10 or Grade 11 did. The relationship between marital status and work-home interaction is not clear, however, as Grzywacz and Marks (2000) reported that being unmarried was associated with negative WHI.

Studies on parental status revealed that the age of children as well as the number of children living at home has an influence on work-home interference in both directions (Grandey \& Cropanzano, 1999; Kunninen \& Mauno, 1998). Grzywacz and Marks (2000) reported that men who have children experienced more positive spill-over from work to home than men without children. Furthermore, Demerouti et al (2004) found that compared to women without children, women with children reported more positive than negative influences from the home domain. With regard to differences between language groups, Pieterse and Mostert (2005) reported no significant differences between language groups.

Other variables investigated in the current study include flexibility at work, if the person has a partner with a paid job and the financial contribution of the partner to the household situation.

\section{2}

\section{Method}

\subsection{Participants and procedure}

A cross-sectional survey design was used to achieve the objectives of this study. Random samples $(n=320)$ were taken from mining houses in the Gauteng, North West and Northern provinces, which included gold, platinum and phosphate mines (response rate $=35$ per cent). Participants included employees of different Patterson grade levels (B2-E2), ranging from employees working underground to managers. Scheduled visits were made to the mining houses. Once permission from management in the participating mining sections had been obtained, the questionnaire was compiled and distributed. A covering letter was included to explain the goal and importance of the study. The letter contained a list of contact persons in case participants had any questions. Participants were assured of the anonymity of their responses and the confidentiality with which the information would be handled. Participants were given three weeks to complete the questionnaires, after which the questionnaires were personally collected by the researchers or sent to the university by the resident HR consultant. Table 1 indicates the characteristics of the participants in the study. 


\section{Table 1}

Characteristics of the participants

\begin{tabular}{|c|c|c|c|}
\hline Item & Category & Frequency & Percentage \\
\hline \multirow[t]{3}{*}{ Gender } & Male & 254 & 79.9 \\
\hline & Female & 64 & 20.1 \\
\hline & Missing values & 2 & 0.6 \\
\hline \multirow[t]{3}{*}{ Ethnicity } & Caucasian & 182 & 56.9 \\
\hline & African & 129 & 40.3 \\
\hline & Missing values & 3 & 0.9 \\
\hline \multirow[t]{5}{*}{ Age } & 22-29 years & 42 & 13.1 \\
\hline & 30-39 years & 126 & 39.4 \\
\hline & 40-49 years & 104 & 32.5 \\
\hline & $50-69$ years & 43 & 13.4 \\
\hline & Missing values & 4 & 1.3 \\
\hline \multirow[t]{4}{*}{ Language } & Afrikaans & 148 & 46.3 \\
\hline & African languages & 128 & 40 \\
\hline & English & 41 & 12.8 \\
\hline & Missing values & 3 & 0.9 \\
\hline \multirow[t]{3}{*}{ Marital status } & Married & 244 & 76.3 \\
\hline & Not married & 73 & 22.7 \\
\hline & Missing values & 3 & 0.9 \\
\hline \multirow[t]{3}{*}{ Education } & Secondary education & 192 & 59.9 \\
\hline & Tertiary education & 122 & 38.1 \\
\hline & Missing values & 6 & 1.9 \\
\hline
\end{tabular}

According to Table 1, the majority of the participants (79.9 per cent) were male, of which 56.9 per cent were Caucasian and 40.3 per cent were African. In total, 148 (46.3 per cent) of the participants were Afrikaans-speaking, with speakers of African languages constituting 128 (40 per cent) of the sample. Only 12.8 per cent were English-speaking. With regard to marital status, 76.3 per cent of the participants were not married (either single or divorced) and 22.7 per cent were married. A total of 192 (59.9 per cent) of the participants had a secondary educational qualification (Grade 12 or lower), while 122 (38.1 per cent) had a tertiary education qualification.

\subsection{Measuring battery}

The following questionnaires were used in the empirical study:

The Survey Work-Home Interaction - NijmeGen (SWING) was used to measure work-home interaction (Geurts et al, 2005). The final version of the SWING used here was a 22-item measure and assessed four types of work-home interference. The first was negative WHI (eight items, for example, "How often does it happen that you do not have the energy to engage in leisure activities with your spouse/family/friends because of your job?"). The second was positive WHI (five items, for example, "How often 
does it happen that you fulfil your domestic obligations better because of the things you have learned on your job?"). The third was negative HWI (four items, for example, "How often does it happen that you have difficulty concentrating on your work because you are preoccupied with domestic matters"). The fourth was positive HWI (five items, for example, "How often does it happen that you take your responsibilities at work more seriously because you are required to do the same at home?"). All items were scored on a four-point frequency rating scale, ranging from " 0 " (never) to "3" (always).

Biographical information was elicited with regard to gender, ethnicity, age, language, qualification, household situation, parental status, as well as the participant's working contract.

\subsection{Statistical analysis}

The statistical analysis was carried out using the SPSS program (SPSS Inc., 2005) and the Amos program (Arbuckle, 2003). Cronbach Alpha coefficients were used to assess the reliability of the scales (Clark \& Watson, 1995). Descriptive statistics (particularly means and standard deviations) were used to analyse the data.

The construct validity of the SWING was tested by comparing four competing models for the relationships among the 22 items, using structural equation modelling (SEM) methods as implemented by Amos (Arbuckle, 2003). The procedure that was followed is explained in the "Results" section of this article. Missing values were replaced with the maximum likelihood estimates of the complete data, as suggested by Little and Rubin (2002). Factorial invariance was examined for the best-fitting factor model using the procedure suggested by Byrne (2001).

Testing for multi-group invariance involved testing for invariance simultaneously across groups (in this case, language and ethnicity), where sets of parameters are put to the test in a logically ordered and increasingly restrictive fashion. Depending on the model and hypotheses to be tested, the following sets of parameters are most commonly of interest in answering questions related to group invariance: firstly, factor loading paths; secondly, factor variances/covariances; and thirdly, structural regression paths. Tests of hypotheses related to group invariance typically begin with scrutiny of the measurement model, where the pattern of factor loadings for each observed measure is tested for its invariance across the groups. Parameters are then constrained equal while subsequent tests of the structural parameters are conducted. As each new set of parameters is tested, those known to be group-invariant are constrained equal.

As a prerequisite for testing for factorial invariance, it is customary to consider a baseline model which is estimated for each group separately. This model represents the one that best fits the data from the perspectives of both parsimony and substantive meaningfulness. Given that the $\chi^{2}$ statistic and its degrees of freedom are additive, the sum of the $\chi^{2}$ values derived from the model-fitting process for each group separately reflects the extent to which the underlying structure fits the data across groups when no cross-group constraints are imposed. Because measuring instruments are often group-specific in the way they operate, baseline models are not expected to be identical across groups. Because the sole use of the $\chi^{2}$ has certain limitations (see Byrne, 2001), researchers have addressed these limitations by developing goodness-of-fit indices that take a more pragmatic approach to the evaluation process.

The following goodness-of-fit-indices were used as adjuncts to the $\chi^{2}$ statistics in this study:

- $\chi^{2} / \mathrm{df}$ ratio;

- the Goodness-of-Fit Index (GFI);

- the Parsimony Goodness-of-Fit Index (PGFI);

- $\quad$ the Incremental Fit Index IFI;

- $\quad$ the Tucker-Lewis Index (TLI);

- the Comparative Fit Index (CFI); and

- the Root Mean Square Error of Approximation (RMSEA).

Hypothesised relationships are tested empirically for goodness-of-fit with the sample data. The $\chi^{2}$ statistic and several other goodness-of-fit indices summarise the degree of correspondence 
between the implied and observed covariance matrices. Jöreskog and Sörborn (1993) suggest that the $\chi^{2}$ value may be considered more appropriately as a badness-of-fit rather than as a goodness-of-fit measure in the sense that a small $\chi^{2}$ value is indicative of good fit. However, because the $\chi^{2}$ statistic equals $(\mathrm{N}-1) \mathrm{F}_{\min }$, this value tends to be substantial when the model does not hold and the sample size is large (Byrne, 2001). A large $\chi^{2}$ relative to the degrees of freedom indicates a need to modify the model to fit the data better. One of the first fit statistics to address this problem was the $\chi^{2} /$ degrees of freedom ratio (CMIN/DF) (Wheaton, Muthén, Alwin \& Summers, 1977). These criteria, commonly referred to as "subjective" or "practical" indices of fit, are typically used as adjuncts to the $\chi^{2}$ statistic.

Multivariate analysis of variance (MANOVA) was used to determine the significance of differences between the work-home interaction levels of different socio-demographic groups. MANOVA tests whether mean differences among groups on a combination of dependent variables are likely to have occurred by chance (Tabachnick \& Fidell, 2001). In MANOVA, a new dependent variable that maximises group differences is created from the set of dependent variables.

Wilk's Lambda was used to test the likelihood of the data under the assumption of equal population mean vectors for all groups, against the likelihood under the assumption that the population mean vectors are identical to those of the sample mean vectors for the different groups. When an effect was significant in MANOVA, one-way analysis of variance (ANOVA) was used to determine which dependent variables had been affected. Because multiple ANOVAs were used, a Bonferroni-type adjustment was made for inflated Type 1 error. The Games-Howell procedure was used to determine whether there were statistical differences between the groups, as recommended by Field (2005).

\section{Results}

\subsection{Confirmatory factor analysis of the SWING}

Following Geurts et al (2005), the construct validity of the SWING was tested with SEM, using the maximum likelihood method. Four competing factorial models were tested. Model 1 (the "one-factor model") proposes that all 22 items load on the same underlying latent dimension, assuming that the items cannot be distinguished on the basis of direction or quality of influence. Model 2 (the "direction model") is a two-factor model, and distinguishes between items that refer to either influence from work or influence from home (irrespective of its quality). Model 3 (the "quality model") also distinguishes between two factors, where the first factor includes all items referring to positive interaction and the second factor includes all items referring to negative interaction (irrespective of the originating domain). Model 4 (the "hypothesised model") represents the four-factor model and distinguishes between the four expected dimensions.

Table 2 presents the fit indices for these models.

Table 2

Goodness-of-fit statistics for the comparison of models

\begin{tabular}{|l|l|c|c|c|c|c|c|c|c|}
\hline \multicolumn{2}{|c|}{ Model } & $\chi^{2}$ & $\chi^{2} / \mathrm{df}$ & GFI & PGFI & IFI & TLI & CFI & RMSEA \\
\hline M1 & One-factor & 1298.26 & 6.21 & 0.62 & 0.54 & 0.56 & 0.51 & 0.55 & 0.13 \\
\hline M2 & $\begin{array}{l}\text { Two-factor } \\
\text { ("Direction model") }\end{array}$ & 1008.56 & 4.85 & 0.71 & 0.59 & 0.68 & 0.64 & 0.67 & 0.11 \\
\hline M3 & $\begin{array}{l}\text { Two-factor } \\
\text { ("Quality model") }\end{array}$ & 805.15 & 3.87 & 0.78 & 0.64 & 0.76 & 0.73 & 0.76 & 0.1 \\
\hline
\end{tabular}




\begin{tabular}{|c|l|c|c|c|c|c|c|c|c|}
\hline M4 & $\begin{array}{l}\text { Four-factor } \\
\text { ("Hypothesised } \\
\text { model") }\end{array}$ & 369.1 & 1.79 & 0.91 & 0.74 & 0.93 & 0.93 & 0.93 & 0.05 \\
\hline M5 & $\begin{array}{l}\text { Four factor } \\
\text { ("Final model") }\end{array}$ & 276.05 & 1.49 & 0.93 & 0.74 & 0.96 & 0.96 & 0.96 & 0.04 \\
\hline
\end{tabular}

From Table 2, it is clear that Model 1 did not fit the data well $\left(\chi^{2}=1298.26_{(n=320)}, d f=209\right.$, $p<0.001$; GFI, AGFI, IFI, TLI and CFI $<0.9$ and RMSEA > 0.08). Model 2 ("the directional model") and Model 3 (the "quality model") explained the associations among the items significantly better than Model 1 (M2 vs. M1: $\Delta$ $\chi^{2}=289.7_{(n=320)}, d f=1, p<0.001 ;$ M3 vs. M1: $\Delta$ $\left.\chi^{2}=493.11_{(n=320)}, d f=1, p<0.001\right)$. However, both these models still fell short of what is acceptable. The four-factor hypothesised model, which distinguished between the four proposed dimensions of work-home interaction, explained the associations among the items significantly better than the other three competing models (M4 vs. M1: $\Delta \chi^{2}=929.16_{(n=320)}, d f=3$, $p<0.001 ;$ M4 vs. M2: $\Delta \chi^{2}=639.46_{(n=320)}$, $d f=2, p<0.001$; M4 vs. M3: $\Delta \chi^{2}=436.055_{(n=}$ $\left.{ }_{320)}, d f=2, p<0.001\right)$.

Inspection of the fit indices of Model 4 suggests a good model fit. However, on inspection of the standardised regression weights, modification indices and standardised residual covariances, one item seems to be problematic ("How often does it happen that after spending a pleasant weekend with your spouse/family/friends, you have more fun in your job?"). In addition, one constrained parameter exhibiting a high degree of misfit lay in the error covariance matrix and represents a correlated error between Item 1 and Item 2 $(\mathrm{MI}=29.65)$. Compared with the MI values for all other error covariance parameters, this value was much higher. Based on these results, Model 4 was re-specified, with the problematic item deleted; and the error between Item 1 and Item 2 was allowed to correlate.

As can be seen from Table 2, Model 5 fitted the data significantly better than M4 (M5 vs. M4: $\Delta \chi^{2}=93.05_{(n=320)}, d f=21, p<$ $0.001)$. Since this model fit was satisfactory and the results agreed with the theoretical assumptions underlying the structure of the SWING, no further modifications of the model were deemed necessary. These results support Hypothesis 1, which postulates that work-home interaction can be characterised as a four-dimensional construct that distinguishes between the direction (work to home, and home to work) and quality (negative and positive) of influence.

\subsection{Factorial invariance of the SWING for language and ethnic groups}

Next, the hypothesis relating to the invariance for factor loadings, factor variances and covariances of the four-factor structure of the SWING was tested for two groups based on language (Afrikaans vs. African Languages) and ethnicity (Caucasian vs. African). At the statistical level, the test for the invariance of factor loading and covariances involves using the $\chi^{2}$ statistics to determine the difference in statistical fit between the unconstrained and the constrained models. Non-significant difference between models indicates statistical support for the hypothesis that is being tested. Invariance can also be examined by comparing the other indices (such as the IFI, TLI, CFI and RMSEA) of the models compared. Such comparisons provide a test for invariance at the practical level, where small differences are indicative of invariance for groups compared. Values of 0.9 and over (for IFI, TLI and CFI) or 0.08 and under (RMSEA) signify an acceptable fit (Byrne, 2001). In general, before testing for measurement and structural invariance, and differences in latent mean scores, it is necessary to ensure well fitting models for the groups involved (Byrne, 2001). Therefore, baseline models were tested for each group. The results are presented in Table 3. 


\section{Table 3}

Testing for invariant factorial structures of the SWING

\begin{tabular}{|c|c|c|c|c|c|c|c|c|}
\hline MODEL & $\chi^{2}$ & $\chi^{2} / \mathrm{df}$ & GFI & PGFI & IFI & TLI & CFI & RMSEA \\
\hline \multicolumn{9}{|l|}{ Language } \\
\hline $\begin{array}{l}\text { Baseline model } \\
\text { (Afrikaans) }\end{array}$ & 271.39 & 1.47 & 0.86 & 0.69 & 0.92 & 0.91 & 0.92 & 0.06 \\
\hline $\begin{array}{l}\text { Baseline model } \\
\text { (African languages) }\end{array}$ & 231.3 & 1.25 & 0.85 & 0.68 & 0.95 & 0.94 & 0.95 & 0.04 \\
\hline Unconstrained model & 502.69 & 1.36 & 0.86 & 0.69 & 0.94 & 0.93 & 0.94 & 0.04 \\
\hline Constrained model & 527.94 & 1.34 & 0.85 & 0.73 & 0.94 & 0.93 & 0.94 & 0.04 \\
\hline \multicolumn{9}{|c|}{$\Delta \chi^{2}=25.25_{(n=320)^{\prime}} d f=25(p<0.01)$} \\
\hline \multicolumn{9}{|l|}{ Ethnicity } \\
\hline $\begin{array}{l}\text { Baseline model } \\
\text { (Caucasian) }\end{array}$ & 293.72 & 1.59 & 0.87 & 0.7 & 0.92 & 0.91 & 0.92 & 0.06 \\
\hline $\begin{array}{l}\text { Baseline model } \\
\text { (African) }\end{array}$ & 231.98 & 1.25 & 0.86 & 0.69 & 0.95 & 0.94 & 0.95 & 0.05 \\
\hline Unconstrained model & 525.73 & 1.42 & 0.86 & 0.70 & 0.93 & 0.92 & 0.93 & 0.04 \\
\hline Constrained model & 557.46 & 1.41 & 0.86 & 0.73 & 0.93 & 0.93 & 0.93 & 0.04 \\
\hline \multicolumn{9}{|c|}{$\Delta \chi^{2}=31.73_{(n=320)^{\prime}} d f=25(p<0.01)$} \\
\hline
\end{tabular}

The results of CFA of the four-factor model showed excellent fit based on language (Afrikaans: $\chi^{2}=271.39_{(n=320)}, d f=1851, p<0.001$; African Languages: $\chi^{2}=231.30_{(n=320)}, d f=$ $1851, p<0.001$ ) as well as ethnicity (Caucasian: $\chi^{2}=293.72_{(n=320)}, d f=1851, p<0.001$; African: $\left.\chi^{2}=231.98_{(n=320)}, d f=1851, p<0.001\right)$. Therefore, these models were used as the baseline models for the language and ethnic groups. Table 3 shows the results of analyses for testing the measurement and structural invariance across language and ethnicity. As can be seen, the practical fit indices of the unconstrained models were very good, supporting the invariance for the number of factors. The indices for the constrained models also showed very good fit, and their values were very close to those for the constrained model. In addition, differences between the models based on the $\chi^{2}$ value were also non-significant $(p<0.01)$. These results provide support for the invariance in the pattern of factor loadings of the SWING across language and ethnicity, providing support for Hypothesis 2.

\subsection{Descriptive statistics, internal consistencies and relationships between the SWING dimensions}

In Table 4, the descriptive statistics, Cronbach Alpha coefficients and correlation coefficients of the SWING are given.

\section{Table 4}

Descriptive statistics, internal consistencies and correlation coefficients of the SWING

\begin{tabular}{|l|l|l|l|l|l|c|}
\hline \multicolumn{1}{|c|}{ Item } & $\mathbf{M}$ & SD & $\alpha$ & $\mathbf{1}$ & $\mathbf{2}$ & $\mathbf{3}$ \\
\hline 1. Negative WHI & 1.14 & 0.67 & 0.9 & - & - & - \\
\hline 2. Positive WHI & 1.46 & 0.63 & 0.74 & 0.06 & - & - \\
\hline 3. Negative HWI & 0.67 & 0.61 & 0.78 & $0.35^{*+}$ & 0.08 & - \\
\hline 4. Positive HWI & 1.66 & 0.77 & 0.77 & $0.14^{*}$ & $0.34^{*+}$ & $0.17^{*}$ \\
\hline
\end{tabular}

* Correlation is statistically significant at the 0.01 level

+ Correlation is practically significant, $r>0.30$ (medium effect) 
From the results set out in Table 4, it can be seen that the relationship between the positive and negative scales of WHI and HWI is highly correlated, as well as statistically and practically significant (medium effect). This would suggest that alteration in one variable would cause a simultaneous and/or congruent alteration in the other. Furthermore, all four scales have acceptable Cronbach Alpha coefficients compared to the guideline of $\alpha \geq 0.70$ (Nunnally $\&$ Bernstein, 1994), providing evidence for the internal consistency of the SWING and support for Hypothesis 3.

\subsection{Differences between socio- demographic groups}

MANOVA (multivariate analysis of variance) was used to determine differences between sociodemographic groups with regard to work-home interaction. Socio-demographic groups included were age, ethnicity, gender, qualification, marital status, parental status, language, flexibility at work, whether the respondent has a partner, and the partner's contribution to the household situation (financially). Results were first analysed for statistical significance using Wilk's Lambda statistics. ANOVA was used to determine specific differences whenever statistical differences were found.

The results of the MANOVA analysis are given below, in Table 5 .

\section{Table 5}

Differences in the work-home interaction levels of socio-demographic groups

\begin{tabular}{|l|c|c|c|c|c|}
\hline \multicolumn{1}{|c|}{ Variable } & Value & $\boldsymbol{F}$ & Df & P & $\begin{array}{c}\text { Partial Eta } \\
\text { squared }\end{array}$ \\
\hline Age & 0.92 & 2.18 & 12 & $0.01^{*}$ & 0.03 \\
\hline Ethnicity & 0.87 & 11.09 & 4 & $0^{*}$ & 0.13 \\
\hline Gender & 0.95 & 3.74 & 4 & $0.01^{*}$ & 0.05 \\
\hline Education & 0.94 & 5.37 & 4 & $0^{*}$ & 0.07 \\
\hline Marital status & 0.96 & 3.57 & 4 & $0.01^{*}$ & 0.04 \\
\hline Parental status & 0.96 & 3.31 & 4 & $0.01^{*}$ & 0.04 \\
\hline Language & 0.87 & 5.62 & 8 & $0^{*}$ & 0.07 \\
\hline Flexibility at work & 0.8 & 4.42 & 16 & $0^{*}$ & 0.05 \\
\hline Has a partner with a paid job & 0.96 & 2.44 & 4 & $0.05^{*}$ & 0.04 \\
\hline $\begin{array}{l}\text { Partners' contribution to the } \\
\text { household situation }\end{array}$ & 0.90 & 1.68 & 12 & 0.07 & 0.04 \\
\hline
\end{tabular}

* $p \leq 0.05=$ significant effect

In an analysis of Wilk's Lambda values, statistically significant differences $(p \leq 0.05)$ regarding work-home interaction levels were found between all the variables, except for the partner's contribution to the household situation. The relationship between workhome interaction and the socio-demographic variables levels that showed a statistically significant difference was analysed further using ANOVA. Because sample sizes were different, the Games-Howell procedure was used to determine whether there were any statistical differences between the groups (see Field, 2005).

The results of the ANOVA based on Age are given below, in Table 6 . 


\section{Table 6}

Differences in work-home interaction levels based on age

\begin{tabular}{|l|c|c|c|c|c|c|}
\hline \multicolumn{1}{|c|}{ Item } & $\mathbf{2 2 - 2 9}$ years & $\mathbf{3 0 - 3 9}$ years & $\mathbf{4 0 - 4 9}$ years & $\mathbf{5 0 - 6 9}$ years & $\boldsymbol{p}$ & $\begin{array}{c}\text { Partial Eta } \\
\text { squared }\end{array}$ \\
\hline Negative WHI & 1.04 & 1.16 & 1.14 & 1.1 & 0.74 & 0 \\
\hline Positive WHI & $1.33^{\mathrm{b}}$ & $1.34^{\mathrm{b}}$ & 1.55 & $1.69^{\mathrm{a}}$ & $0^{*}$ & 0.04 \\
\hline Negative HWI & 0.54 & 0.76 & 0.61 & 0.64 & 0.14 & 0.02 \\
\hline Positive HWI & 1.4 & 1.72 & 1.65 & 1.76 & 0.09 & 0.02 \\
\hline
\end{tabular}

* Statistically significant difference: $p \leq 0.05$

${ }^{a}$ Group differs statistically significantly from type (in row) where ${ }^{b}$ is indicated

Table 6 shows that statistically significant differences exist between levels of positive WHI based on age. It appears that participants aged between 50 and 69 years experienced statistically significantly higher levels of positive WHI, compared to the age groups of 22-29 years and 30-39 years.

The results of the ANOVA based on Ethnicity are given in Table 7.

Table 7

Differences in work-home interaction levels based on ethnicity

\begin{tabular}{|l|c|c|c|c|}
\hline \multicolumn{1}{|c|}{ Item } & Caucasian & African & $\boldsymbol{p}$ & $\begin{array}{c}\text { Partial Eta } \\
\text { squared }\end{array}$ \\
\hline Negative WHI & 1.1 & 1.18 & 0.27 & 0 \\
\hline Positive WHI & 1.31 & 1.66 & $0^{*}$ & 0.07 \\
\hline Negative HWI & 0.59 & 0.77 & $0^{*}$ & 0.02 \\
\hline Positive HWI & 1.49 & 1.93 & $0^{*}$ & 0.08 \\
\hline
\end{tabular}

* Statistically significant difference: $p \leq 0.05$

Table 7 shows that statistically significant differences exist between Caucasians and Africans in terms of positive WHI, negative HWI and positive HWI. African participants experienced higher positive WHI and positive HWI, but also had higher negative HWI levels than Caucasian participants had.

The results of the ANOVA based on Gender are given below, in Table 8 .

\section{Table 8}

Differences in work-home interaction levels based on gender

\begin{tabular}{|l|c|c|c|c|}
\hline \multicolumn{1}{|c|}{ Item } & Male & Female & $\boldsymbol{p}$ & Partial Eta squared \\
\hline Negative WHI & 1.19 & 0.91 & $0^{*}$ & 0.03 \\
\hline Positive WHI & 1.50 & 1.3 & $0.03^{*}$ & 0.02 \\
\hline Negative HWI & 0.7 & 0.52 & $0.03^{*}$ & 0.01 \\
\hline Positive HWI & 1.69 & 1.56 & 0.22 & 0 \\
\hline
\end{tabular}

* Statistically significant difference: $p \leq 0.05$ 
Table 8 shows that statistically significant differences exist between males and females based on negative WHI, positive WHI and negative HWI. Based on these results, it seems that males experienced higher levels of negative WHI and negative HWI, but also higher levels of positive WHI. However, the ratio of male to female participants should be noted (males 79.9 per cent and females 20.1 per cent).

Differences between educational groups are presented in Table 9.

Table 9

Differences in work-home interaction levels based on qualification

\begin{tabular}{|l|c|c|c|c|}
\hline \multicolumn{1}{|c|}{ Item } & $\begin{array}{c}\text { Secondary } \\
\text { education }\end{array}$ & Tertiary education & $\boldsymbol{p}$ & $\begin{array}{c}\text { Partial Eta } \\
\text { squared }\end{array}$ \\
\hline Negative WHI & 1.23 & 0.99 & $0^{*}$ & 0.03 \\
\hline Positive WHI & 1.44 & 1.48 & 0.59 & 0 \\
\hline Negative HWI & 0.73 & 0.58 & $0.03^{*}$ & 0.02 \\
\hline Positive HWI & 1.77 & 1.51 & $0^{*}$ & 0.03 \\
\hline
\end{tabular}

* Statistically significant difference: $p \leq 0.05$

Table 9 shows that statistically significant differences exist between individuals with a secondary education compared to those with a tertiary education. Individuals who had a tertiary education appeared to experience lower levels of negative WHI, as well as lower levels of negative and positive HWI. Therefore, individuals who had a secondary education scored higher on negative WHI and HWI, but at the same time appeared to experience more positive HWI than participants possessing a tertiary education.

The results of the ANOVA based on Marital and Parental Status are given in Table 10 and Table 11 respectively.

Table 10

Differences in work-home interaction levels based on marital status

\begin{tabular}{|l|c|c|c|c|}
\hline \multicolumn{1}{|c|}{ Item } & Married & Unmarried & $\boldsymbol{p}$ & $\begin{array}{c}\text { Partial Eta } \\
\text { squared }\end{array}$ \\
\hline Negative WHI & $1.18^{\mathrm{b}}$ & $0.97^{\mathrm{a}}$ & $0.02^{*}$ & 0.02 \\
\hline Positive WHI & $1.51^{\mathrm{b}}$ & $1.3^{\mathrm{a}}$ & $0.01^{*}$ & 0.02 \\
\hline Negative HWI & 0.66 & 0.7 & 0.62 & 0 \\
\hline Positive HWI & 1.69 & 1.56 & 0.21 & 0.01 \\
\hline
\end{tabular}

* Statistically significant difference: $p<0.05$

a Group differs statistically significantly from type (in row) where $b$ is indicated

Statistically significant differences were found between married and unmarried participants, with married participants experiencing higher levels of positive and negative WHI. However, no statistically significant differences were found between negative and positive HWI. 


\section{Table 11}

Differences in work-home interaction levels based on parental status

\begin{tabular}{|l|c|c|c|c|}
\hline \multicolumn{1}{|c|}{ Item } & With children & Without children & $\boldsymbol{p}$ & $\begin{array}{c}\text { Partial Eta } \\
\text { squared }\end{array}$ \\
\hline Negative WHI & 1.17 & 0.96 & $0.02^{*}$ & 0.02 \\
\hline Positive WHI & 1.48 & 1.34 & 0.11 & 0.01 \\
\hline Negative HWI & 0.68 & 0.56 & 0.15 & 0.01 \\
\hline Positive HWI & 1.73 & 1.41 & $0^{*}$ & 0.03 \\
\hline
\end{tabular}

* Statistically significant difference: $p \leq 0.05$

In Table 11, the ANOVA results show that statistically significant differences were found between employees with children and those without children with regard to negative WHI and positive HWI. Individuals with children appeared to have higher levels of negative WHI than those without children, but at the same time also experienced higher levels of positive HWI.

Table 12

Differences in work-home interaction levels based on language

\begin{tabular}{|l|c|c|c|c|c|}
\hline \multicolumn{1}{|c|}{ Item } & Afrikaans & English & African & $\boldsymbol{p}$ & $\begin{array}{c}\text { Partial Eta } \\
\text { squared }\end{array}$ \\
\hline Negative WHI & 1.11 & 1.01 & 1.19 & 0.3 & 0.01 \\
\hline Positive WHI & $1.32^{\mathrm{b}}$ & $1.36^{\mathrm{b}}$ & $1.65^{\mathrm{a}}$ & $0^{*}$ & 0.07 \\
\hline Negative HWI & $0.6^{\mathrm{a}}$ & 0.59 & $0.78^{\mathrm{b}}$ & $0.03^{*}$ & 0.02 \\
\hline Positive HWI & $1.54^{\mathrm{a}}$ & 1.3 & $1.92^{\mathrm{b}}$ & $0^{*}$ & 0.09 \\
\hline
\end{tabular}

* Statistically significant difference: $p \leq 0.05$

a Group differs statistically significantly from type (in row) where ${ }^{b}$ is indicated

As noted in Table 12, African-speaking individuals experienced statistically significantly higher levels of positive WHI and HWI than Afrikaans- and English-speaking participants did. However, at the same time, Africanspeaking individuals experienced higher levels of negative HWI.
Table 13 shows the differences in workhome interaction based on Flexibility at work. Differences are determined based on the possibility to take a day off from work or to work from home when something unexpected happens at home (for example, when a child gets ill or a repair person comes to the house).

Table 13

Differences in work-home interaction levels based on flexibility at work

\begin{tabular}{|l|c|c|c|c|c|c|c|}
\hline \multicolumn{1}{|c|}{ Item } & $\begin{array}{c}\text { Very easy } \\
\text { to arrange }\end{array}$ & $\begin{array}{c}\text { Easy to } \\
\text { arrange }\end{array}$ & $\begin{array}{c}\text { Possible to } \\
\text { arrange }\end{array}$ & $\begin{array}{c}\text { May not be } \\
\text { possible to } \\
\text { arrange }\end{array}$ & $\begin{array}{c}\text { Impossible } \\
\text { to arrange }\end{array}$ & $\begin{array}{c}p \\
\text { squartial Eta }\end{array}$ \\
\hline Negative WHI & $0.76^{\mathrm{a}}$ & $0.9^{\mathrm{c}}$ & $1.09^{\mathrm{be}}$ & $1.5^{\mathrm{bdf}}$ & $1.77^{\mathrm{bdf}}$ & $0^{*}$ & 0.17 \\
\hline Positive WHI & 1.6 & 1.46 & 1.41 & 1.45 & 1.52 & 0.61 & 0.01 \\
\hline Negative HWI & 0.5 & 0.64 & 0.7 & 0.63 & 0.77 & 0.43 & 0.01 \\
\hline Positive HWI & 1.62 & 1.57 & 1.68 & 1.6 & 1.84 & 0.63 & 0.01 \\
\hline
\end{tabular}

* Statistically significant difference: $p \leq 0.05$

a Group differs statistically significantly from type (in row) where ${ }^{\mathrm{b}}$ is indicated; ${ }^{\mathrm{c}}$ Group differs statistically significantly from type (in row) where ${ }^{\mathrm{d}}$ is indicated; ${ }^{\mathrm{e}}$ Group differs statistically significantly from type (in row) where ${ }^{f}$ is indicated 
As can be seen in Table 13, statistically significant differences were found between groups with regard to negative WHI. Participants who found it very easy or easy to arrange a work schedule experienced statistically significantly lower negative WHI than those who found it possible, difficult or impossible to arrange.

The results of the ANOVA based on whether a respondent had a partner with a paid job are given below in Table 14 .

\section{Table 14}

Differences in work-home interaction levels based on having a partner with/without a paid job

\begin{tabular}{|l|c|c|c|c|}
\hline \multicolumn{1}{|c|}{ Item } & $\begin{array}{c}\text { Partner with a } \\
\text { paid job }\end{array}$ & $\begin{array}{c}\text { Partner without a } \\
\text { paid job }\end{array}$ & $\boldsymbol{p}$ & $\begin{array}{c}\text { Partial Eta } \\
\text { squared }\end{array}$ \\
\hline Negative WHI & 1.09 & 1.23 & 0.1 & 0.01 \\
\hline Positive WHI & 1.47 & 1.43 & 0.56 & 0 \\
\hline Negative HWI & 0.58 & 0.75 & $0.03^{*}$ & 0.02 \\
\hline Positive HWI & 1.56 & 1.74 & 0.06 & 0.01 \\
\hline
\end{tabular}

* Statistically significant difference: $p<0.05$

Table 14 shows that statistically significant differences occurred between individuals who had a partner with a paid job and those whose partner did not have a paid job with regard to negative HWI. It seems that participants without a partner with a paid job experienced statistically significant higher negative HWI than those with a partner with a paid job.

\section{Discussion}

The aim of this study was to build on existing findings with regard to the application of the SWING in order to provide evidence of the applicability of this instrument in the mining industry. In addition, valuable information was added to the literature with regard to the relationship between work-home interaction and socio-demographic characteristics.

Regarding the construct validity of the SWING, four competing factorial models were tested. The results indicated that the four-factor hypothesised model fitted the data significantly better than the alternative models. However, the fit indices, factor loadings and modification indices indicated that the four-factor model could be improved by deleting one problematic item ("How often does it happen that after spending a pleasant weekend with your spouse/ family/friends, you have more fun in jour job?”) and allowing a correlated error between two items (Items 1 and 2). It is possible that this item was difficult for some of the participants to understand. A possible explanation for the covariation between the two item errors could be that items with comparable rating scales often have measurement errors that are correlated (Byrne, 1989).

After these modifications were made, the fit of the hypothesised model to the data was satisfactory, confirming that work-home interaction can be characterised as a fourdimensional construct that distinguishes between the direction (work to home, and home to work) and quality (negative and positive) of influence. This confirms the results of Geurts $e t$ al (2005), whose results showed that the SWING questionnaire reliably measured four empirically distinct types of work-home interaction. It also supports the findings of Pieterse and Mostert (2005) and Rost and Mostert (2007), who also confirmed a four-factor structure.

Although the factor structure of the SWING was confirmed, it could not be assumed that this structure was invariant for the different groups. Because South Africa contains many different language and ethnic groups, it was decided to test the factorial invariance of the four-factor structure for two language groups (Afrikaans and African languages) and for two ethnic groups 
(Caucasian and African). The results showed that the four-factor structure was invariant for both these groups, indicating that the instrument measures the same construct for both language and ethnic groups. These results support the findings of Pieterse and Mostert (2005), who also demonstrated construct equivalence for two language groups. Furthermore, acceptable Cronbach Alpha coefficients were obtained for all four scales. This indicates that the SWING is also a reliable instrument to measure workhome interaction and supports previous findings (e.g. Geurts et al, 2005; Pieterse \& Mostert, 2005; Rost \& Mostert, 2005).

With regard to the work-home interaction between socio-demographic groups, statistically significantly differences were found based on age, ethnicity, gender, education, marital status, parental status, language, flexi-bility at work and individuals who have a partner with a paid job.

With regard to age, it was found that participants between the ages of 50 and 69 years experienced statistically significant higher levels of positive WHI, while participants between the ages of 22 and 39 experienced the lowest levels of positive WHI. These findings are congruent with those of Grzywacz and Marks (2000). A possible explanation could be that older individuals may have acquired the necessary skills to manage the demands or conflicts of both domains. It could also prove useful to investigate the activities that older people engaged in, as they may prove helpful in alleviating the pressures of their environments. A possible reason for younger employees experiencing lower levels of positive WHI could be the fact that they are largely inexperienced and, as a result, feel unsettled in themselves and their work (Grzywacz \& Marks, 2000).

The results showed that Africans experience higher levels of positive WHI, as well as more negative WHI and HWI. This contradicts previous results (Grzywacz \& Marks, 2000; Pieterse \& Mostert, 2005). Possible reasons for this finding may be that each culture has distinguishing attributes and these affect the way in which they interpret situations and circumstances. Differences were also found between language groups, where individuals speaking African languages experienced statistically significant higher levels of positive WHI and positive HWI compared to Afrikaans- and English-speaking participants. It should be borne in mind ethnic groups are not equally distributed across all levels in the mines and that the job type or working conditions could be different for the two groups. In general, African participants make up the largest proportion of workers at the lowest levels (usually employees working underground), where Caucasians are predominantly in management positions, which could also influence their work-home interaction in different ways.

Significant differences were found between males and females, where males experience higher levels of negative WHI and negative HWI, but also higher levels of positive WHI. This is in contrast with previous findings, where no differences were found based on gender (Burke, 1988; Demerouti et al, 2004; Eagle et al, 1997; Frone, 2002; Kinnunen \& Mauno, 1998; Kirchmeyer, 1992). However, the ratio of male to female participants should be noted (males 79.9 per cent and females 20.1 per cent). These results should therefore be interpreted with caution.

Differences based on qualifications indicated that participants who had a tertiary education experienced overall lower levels of negative WHI and negative HWI, as well as lower positive HWI. This supports the findings of Pieterse and Mostert (2005) and Van Tonder (2005). However, people with higher qualifications also experienced higher positive WHI. This could be due to the fact that these individuals have more resources and more support, and that they are in positions to learn new skills and become skilled at how to prioritise their family life above that of work.

Statistically significant differences were found between married and unmarried participants, with married participants experiencing higher levels of positive and negative WHI. This is in contrast to the findings of Grzywacz and Marks (2000), who found that unmarried people experienced more negative WHI. Again, the ratio of married to unmarried participants should be noted (married 76.3 per cent versus unmarried 22.7 per cent). These results should therefore also be interpreted with caution. 
Regarding parental status, working parents experienced higher levels of negative WHI than those without children, but at the same time also experienced higher levels of positive HWI. These results are in line with those of Grzywacz and Marks (2000), as well as with those reported by Demerouti et al (2004).

Finally, with regard to flexibility at work, participants would appear to have difficulty in adjusting their work to compensate for permanent home demands and/or situations that may arise. According to Schaufeli and Bakker (2004), autonomy plays an extrinsic motivational role by being instrumental in achieving work goals. Autonomy can also be considered a resource which is purposeful in achieving work goals, reduces job demands and encourages personal growth and development. Therefore, when there is a lack of autonomy, individuals may find it more difficult to combine work and home demands, causing a negative interaction between these two domains.

\section{5}

\section{Limitations and recommendations}

A number of limitations of the present study should be noted. Firstly, the results were obtained exclusively through the use of selfreport questionnaires, which may have created "method-variance" or "nuisance". Secondly, although the results confirmed those of previous findings - strengthening the assumption that the SWING is a valid, invariant and reliable instrument to measure work-home interaction - these results are only applicable to the mining industry. One should therefore be careful to generalise the findings to other occupational groups, particularly to ones outside the manufacturing industry. A third limitation to this study is that organisations such as the mining industry often have unique characteristics such as organisational-specific cultures and/or norms that may have had an impact on the participants' responses. Finally, for some socio-demographic groups the sub-group sizes were not equal (in terms of gender, age, marital status). Since these results could be misleading, one should be careful to interpret the findings where these groups were included.
Based on the results of the present study, as well as on those of Pieterse and Mostert (2005) and Rost and Mostert (2007), the SWING is recommended in assessing work-home interaction within South Africa. The four scales of the SWING can be used to provide useful information on the interaction between work and home. Problematic items could be reformulated to avoid misunderstanding and enhance understanding. Naturally, it would be advantageous to get the SWING translated into the official African languages. The results of this study also provide some useful information on how certain socio-demographic groups differ in their experiences of work and private lives, which could be a starting point for deciding where to focus necessary attention.

In general, the findings of this study suggest that there are indeed differences between socio-demographic groups with regard to their experiences of work-home interaction. As a result, men, younger and older workers (consistent with those studying or caring for elderly dependants), those without children or older children, those from outside the main ethnic groups and those in lower status occupational groups may not be catered for by current work-life balance approaches. While further research on the demographic relationships with aspects of work-life balance and culture in organisations is needed, the findings of this study suggest that there is a need to move the work-life balance agenda beyond the traditional focus (women with young children and white-collar professional and managerial workers).

As Tsui and Gutek (1999) emphasise, demography is complex. Demographic attributes are multiple and interdependent and may mean different things to different people in different situations and results may vary between units of analysis. Since organisations are dynamic social systems, cross-sectional studies may be misleading. Therefore, demographic effects must incorporate the influence of context in situ. Future research should use longitudinal and cross-level studies that examine different demographic dimensions and multidimensional patterns of demography. 
Finally, although one can speculate about possible explanations for group differences, these findings are exploratory in nature and indicative only. Further research is needed before drawing conclusions on these effects.

\section{References}

ALLEN, TD; HERST, DE; BRUCK, CS \& SUTTON, M (2000) "Consequences associated with workto-family conflict: A review and agenda for future research", Journal of Occupational Health Psychology, 5: 278-308.

ARBUCKLE, JL (1999) Amos 5.0. Smallwaters:

Chicago, IL.

BADENHORST, JCC \& VAN SCHALKWYK, SJ (1992) "Minimizing post traumatic stress in critical mining incidents", in Maiden, R.P. (ed.) Employee Assistance Programs in South Africa. Haworth: New York.

BURKE, RJ (1988) "Some antecedents and consequences of work-family conflict", Journal of Social Behavior and Personality, 3: 287-302. BYRNE, BM (1989) A Primer of LISREL: Basic Applications and Programming for Confirmatory Factor Analysis Models. Springer: New York.

BYRNE, BM (2001) Structural Equation Modelling with Amos: Basic Concepts, Applications and Programming. Lawrence Erlbaum: Mahwah, NJ..

CALITZ, PL (2004) The experience of women in the platinum mining industry. Unpublished Master's dissertation. North-West University, Potchefstroom. CARLSON, DS; KACMAR, MK; WAYNE, JH \& GRZYWACZ, JG (2006) "Measuring the positive side of the work-family interface: Development and validation of a work-family enrichment scale", Journal of Vocational Behavior, 68(1): 131-164.

CHAMBER OF MINES OF SOUTH AFRICA (1989)

Mine Safety Division, Mining Survey. Johannesburg. CLARK, LA \& WATSON, D (1995) "Constructing validity: Basic issues in objective scale development", Psychological Assessment, 7: 309-319.

DEMEROUTI, E; GEURTS, SAE; BAKKER, AB \& EUWEMA, M (2004) "The impact of shiftwork on work-home conflict, job attitudes and health", Ergonomics, 47(9), July: 987-1002.

DRENTH, PJD; THIERRY, H \& DE WOLFF, CJ (eds.) (1998) Handbook of Work and Organizational Psychology (2nd ed.) Psychology Press: Hove.

EAGLE, BW; MILES, EW \& ICENOGLE, ML (1997)

"Interrole conflicts and the permeability of work and family domains: Are there gender differences?", Journal of Vocational Behavior, 50: 168-184.
ELDRIDGE, R \& MILES, A (2008) "Demographics research: Summary and implications”, http://www. talentdrain.com (Accessed 3 October 2006).

FIELD, A (2005) Discovering Statistics Using SPSS (2 $2^{\text {nd }}$ ed.) Sage: London.

FRONE, MR (2002) "Work-family balance", in Quick, JC \& Tetrick, LE (eds.) Handbook of Occupational Health Psychology (pp. 143-162). American Psychological Association: Washington, DC. FRONE, MR; RUSSELL, M \& COOPER, ML (1997) "Relation of work-family conflict to health outcomes: A four-year longitudinal study of employed parents", Journal of Occupational and Organizational Psychology, 70: 325-335.

GEURTS, SAE; KOMPIER, MAJ; ROXBURGH, S \& HOUTMAN, ILD (2003) "Does work-home interference mediate the relationship between workload and well-being?", Journal of Vocational Behavior, 63(3): 532-559.

GEURTS, SAE; TARIS, TW; KOMPIER, MAJ; DIKKERS, JSE; VAN HOOFF, MLM \& KINNUNEN, UM (2005) "Work-home interaction from a work psychological perspective: Development and validation of a new questionnaire, the SWING", Work \& Stress, 19(4): 319-339.

GRANDEY, A \& CROPANZANO, R (1999)

"The conservation of resources model applied to work-family conflict and strain", Journal of Vocational Behavior, 54: 350-370.

GREENHAUS, JH \& BEUTELL, NJ (1985) "Sources of conflict between work and family roles", Academy of Management Review, 10: 76-88.

GRZYWACZ, JG \& MARKS, NF (2000)

"Reconceptualizing the work-family interface: An ecological perspective on the correlates of positive and negative spillover between work and family", Journal of Occupational Health Psychology, 5(1): 111-126.

HOUSTON, DM (2005) Work-life Balance in the 21 $1^{\text {st }}$ Century. Palgrave Macmillan: New York.

JÖRESKOG, KG \& SÖRBORN, D (1993) LISREL 8: Structural equation modeling with the SIMPLIS command language. Hillsdale, NJ: Lawrence Erlbaum Associates.

KINNUNEN, U \& MAUNO, S (1998) "Antecedents and outcomes of work-family conflict among employed women and men in Finland", Human Relations, 51: 157-177.

KIRCHMEYER, C (1992) "Perceptions of nonworkto-work spillover: Challenging the common view of conflict-ridden domain relationships", Basic and Applied Psychology, 13: 231-249.

LEWIS, S \& COOPER, CL (2005) Work-life Integration Case Studies of Organisational Change. Wiley: Chichester. 
LITTLE, RA \& RUBIN, DB (2002) Statistical Analysis with Missing Data ( $2^{\text {nd }}$ ed.). Wiley: Hoboken. MCGWIN, G (JNR); VALENT, F; TAYLOR, AJ; HOWARD, HJ; DAVIS, GG; BRISSIE, RM \& RUE ILL, LW (2002) Nov "Epidemiology of fatal occupational injuries in Jefferson County, Alabama", Southern Medical Journal, 95(11): 1300-1312.

MEIJMAN, TF \& MULDER, G (1998) "Psychological aspects of workload", in Drenth, PJ; Thierry, H \& De

Wolff, CJ (eds.) Handbook of Work and Organizational Psychology ( $2^{\text {nd }}$ ed.)

(pp. 5-33) Erlbaum: Hove.

NUNNALLY, JC \& BERNSTEIN, IH (1994)

Psychometric Theory ( $3^{\text {rd }}$ ed.). McGraw-Hill: New York. PARASURAMAN, S \& GREENHAUS, JH (1999)

Integrating Work and Family: Challenges for a Changing

World. Praeger: Westport, CT.

PIETERSE, M \& MOSTERT, K (2005) “Measuring the work-home interface: Validation of the Survey Work-Home Interaction - (SWING) Instrument", Management Dynamics, 14(2): 2-15.

ROST, I \& MOSTERT, K (2007) “The interaction between work and home of employees in the earthmoving equipment industry: Measurement and prevalence", South African Journal of Industrial Psychology, 33(2): 54-61.

SCHAUFELI, WB \& BAKKER, AB (2004) “Job demands, job resources, and their relationship with burnout and engagement: A multi-sample study", Journal of Organizational Behavior, 25(3): 293-315. SINGER, R (2002) "South African women gain ground below surface”, USA Today, May: 1-2. SLUITER, JK (1999) How about work demands, recovery, and health. Unpublished Doctoral thesis, University of Amsterdam, the Netherlands. SPSS Inc. (2003) SPSS 12.0 for Windows. SPSS Inc.: Chicago, IL.

TABACHNICK, BG \& FIDELL, LS (2001) Using Multivariate Statistics ( $4^{\text {th }}$ ed.). Allyn \& Bacon: Boston, MA.

TSUI, AS \& GUTEK, BA (1999) Demographic Differences in Organizations: Current Research and Future Directions. Lexington Books: Lanham, MD. URSIN, H (1980) "Personality, activation and somatic health: A new psychosomatic theory", in Levine, S. \& Ursin, H. (eds.), Coping and Health (pp. 259-279). Plenum: New York.

VAN TONDER, HP (2005) A Psychometric Analysis of the Survey Work-Home Interaction Nijmegen (SWING) in a Nursing Environment. Unpublished Master's dissertation, North West University, Potchefstroom Campus, South Africa.

WHEATON, B (1983) "Stress, personal coping resources, and psychiatric symptoms: An investigation of interactive models", Journal of Health and Social Behavior, 24: 208-229. 